\title{
An in silico analysis of drugs potentially modulating the cytokine storm triggered by SARS-CoV-2 infection
}

\author{
Laura Sanchez-Burgos¹, Gonzalo Gómez-López², Fátima \\ Al-Shahrour ${ }^{2}$ \& Oscar Fernandez-Capetillo ${ }^{1,3^{*}}$
}

${ }^{1}$ Genomic Instability Group, Spanish National Cancer Research Centre, Madrid 28029, Spain 2Bioinformatics Unit, Spanish National Cancer Research Centre, Madrid 28029, Spain ${ }^{2}$ Science for Life Laboratory, Division of Genome Biology, Department of Medical Biochemistry and Biophysics, Karolinska Institute, S-171 21 Stockholm, Sweden

Correspondence should be addressed to O.F. (ofernandez@cnio.es); Twitter: @KP_Twitt_llo

KEYWORDS: SARS-CoV-2; CMap; Cytokine Storm; NF-kB; Glucocorticoids, MEK, Estrogens. 


\section{SUMMARY}

The ongoing COVID-19 pandemic is one of the biggest health and societal challenges of the recent decades. Among the causes of mortality triggered by SARS-CoV-2 infection, the presence of an inflammatory "cytokine storm" (CS) at later stages of the disease has been found to play a determinant role. Here, we used available transcriptomic data from the bronchoalveolar lavage fluid (BALF) of COVID-19 patients suffering from a CS to obtain gene-signatures associated to this pathological process. Using these signatures, we interrogated the Connectivity MAP (CMap) dataset that contains the effects of over 5,000 small molecules on the transcriptome of human cancer cell lines, and looked for molecules which effects on transcription mimic or oppose those associated to the CS. Consistent with their medical use, this analysis found a significant enrichment of glucocorticoids or inhibitors of the Janus Kinases (JAK) as drugs that could revert the CS. On the other hand, molecules that potentiate the immune response such as PKC activators are predicted to worsen the CS. Besides these expected findings, our analysis also reveals a potential effect of the antibiotic doxycycline or MAPK/RAF/MEK inhibitors in reverting the CS, or of topoisomerase inhibitors and the anti-alcohol abuse drug disulfiram in potentiating its effects. Finally, our analyses support that the gender-related differences in the severity of COVID-19 are related to the anti-inflammatory properties of female hormones. While acknowledging that this is an analysis based on limited available data, we decided to share it as a resource that might help others in the selection of drugs that could be tested in the context of experimental models of CS triggered by viral infections. 


\section{INTRODUCTION}

Since the first patient was hospitalized in Wuhan on December $12^{\text {th }} 2019$ (1), the ongoing COVID-19 pandemic has caused 6,288,771 confirmed infections and 379,941 deaths by June $3^{\text {rd }} 2020$ (2). The disease-causing pathogen is SARS-CoV-2, a novel positive sense single-stranded RNA coronavirus belonging to the lineage $B$ of the betacoronavirus genus (3). Infections by different coronavirus strains have been historically present in humans, although they most often only cause a mild cold (e.g. infections by HKU, 229 and hCOV-OC43 coronaviruses) (4). However, several new coronavirus strains have emerged in the last 2 decades that cause an Acute Respiratory Distress Syndrome (ARDS) with severe health consequences including the patients' death. These include SARS-CoV, which between 2002 and 2003 led to 8,000 cases with a mortality of $9.5 \%$ and MERS-CoV, which in 2012 caused 2,500 confirmed infections with a mortality rate of $36 \%(5)$. As of today, and while there is significant variability between countries and final data are still to be fully defined, the averaged worldwide mortality associated to SARS-CoV-2 infections is around 6\% (6).

Due to the lack of a curative treatment for COVID-19, overwhelming efforts are being dedicated to the development therapies including vaccines (7), plasma transfusions from convalescent patients (8) and antiviral treatments limiting viral replication (9) or infection $(10,11)$. Nevertheless, and while respiratory failure associated to ARDS is the leading cause of mortality in COVID-19 patients, accumulating evidence shows that the lethality in a subgroup of the severe patients occurs due to the late onset of an inflammatory "cytokine storm" (CS) (12). First defined in 1993 in the context of graft-versus-host disease (13), the CS has been observed in a wide range of inflammatory diseases such as multiple sclerosis or pancreatitis, and in the context of viral infections including SARS-CoV $(14,15)$. In COVID-19, the CS is caused by an overproduction of proinflammatory cytokines such as IL-6, IL-1 $\beta$ and TNF $\alpha$ by lung infiltrating alveolar macrophages, which triggers vascular hyperpermeability, multiorgan failure and ultimately cell death (16). Potential treatments for the CS of COVID-19 patients include broad spectrum anti-inflammatory therapies such as corticosteroids or more targeted therapies like anti-IL-6 receptor antibodies (Tocilizumab) (17). Other therapies like Janus Kinase (JAK) or IL-1 inhibitors have 
also been proposed as treatments for the CS based on their potent effects in suppressing key inflammatory signaling pathways driven by IFN $\gamma$ or NFKB, respectively, and are being evaluated in clinical trials (18). Importantly, the use of antiinflammatory therapies such as corticosteroids is only recommended in the severe cases undergoing a CS and not in mild cases of COVID-19, as the use of antiinflammatory therapies in earlier stages of the disease would limit the efficacy of the immune system in fighting the infection.

Databases containing transcriptional signatures of human cancer cell lines exposed to a specific perturbation (perturbagen) such as drugs or genetic manipulations have become an excellent platform for data mining in biomedical research (19-21). For instance, the Connectivity Map (CMap) from the Broad Institute at MIT stores over $1,5 \mathrm{M}$ signatures from a wide range of human cancer cell lines exposed to around 5,000 drugs and 3,000 genetic perturbations (overexpression or shRNA-mediated depletion) (https://www.broadinstitute.org/connectivity-map-cmap) (22, 23). These signature databases can be used, for instance, to identify the mechanism of action of a drug, by comparing the transcriptional signature induced by the compound to that induced by genetic perturbation of an individual gene (23). Another relevant use of CMap is to identify drugs that induce a transcriptional signature which negatively correlates with that associated to a given disease as a strategy for discovering potential new therapies (24). In the context of the COVID-19 pandemic, the CMap has been recently used to identify drugs capable of modifying the levels of ACE2 (25), the receptor that SARS-CoV-2 uses for viral entry (10). Here, and based on recently available transcriptomic data from COVID-19 patients, we used CMap to identify drugs that could potentially alleviate or aggravate the severity of the CS associated to late stages of this disease. 


\section{RESULTS AND DISCUSSION}

\section{Biological pathways related to the hypercytokinemia found in COVID-19 patients}

In order to define a transcriptional signature related to COVID-19, we used recently published transcriptomics data from bronchoalveolar lavage fluid (BALF) cells where COVID-19 patients $(n=8)$ were compared to healthy individuals $(n=20)$ and community-acquired pneumonia patients $(n=146)(26)$. Consistent with clinical observations, this study found that COVID-19 patients present a distinct activation of an IFN-dependent cytokine response. We thus defined a transcriptional signature based on the cytokines and cytokine receptor genes that were differentially expressed in COVID-19 patients (COVIDCS) (signature available in Table S1). Using this signature and the clue.io tool from CMap (http://clue.io) we first looked into biological pathways (CMap classes) that have transcriptional signatures that correlate with the COVID ${ }^{\mathrm{CS}}$. Consistent with their key roles in inflammation (27), these analyses identified activation of $\mathrm{NF} \kappa \mathrm{B}$ and $\mathrm{PKC}$ signaling as the CMap classes most significantly similar to the COVIDCS (Fig. 1A and Table S2). Of note, PKC activation triggers NFKB and TNF $\alpha$-dependent inflammatory responses in human bronchial epithelial cells, suggesting that PKC could act early on in triggering the inflammatory response (28). Furthermore, interrogation of CMap for signatures arising from overexpressed genes identifies factors related to inflammation such as IL1 (IL1R1) or TNF $\alpha$ receptors (TNFRS10A and TNFRSF1A) and IFN-response genes (IRF2 and IFR5) as those that most significantly mimic the signature of the COVID ${ }^{\mathrm{CS}}$ (Fig. 1B and Table S3). Thus, as expected, the transcriptional signature of the hypercytokinemia triggered by SARSCoV-2 infection resembles that of activated inflammatory pathways regulated by PKC, $N F \kappa B, T N F \alpha$ and IL1.

In what regards for genes that, when downregulated, trigger a signature similar to the COVIDCS the list was not as clearly restricted to immune factors as the one from overexpressed genes, although it included anti-inflammatory factors such as NFKB inhibitor alpha (NFKBIA) (Fig. 1C and Table S4). Interestingly, Gene Ontology (GO) analyses of genes whose transcriptional signature opposes that of the COVIDCS detected an enrichment in pathways related to female sexual hormones (Fig. 1D). We find this of interest since even if male and females are equally infected with SARS- 
CoV-2 the fatality rate is significantly higher in men, which could be related to genderrelated differences in the intensity of inflammatory responses (29). In fact, several studies have indicated that estrogen levels influence the severity of diseases linked to inflammation, including cancer, due the modulation of NFКB signaling (30-33). Moreover, gender differences in COVID-19 mortality rates are highest in younger patients and decline progressively, further supporting a connection with female hormone levels (34). Noteworthy, estrogen treatments are already been explored in clinical trials for reducing the severity of COVID-19 in men and women 55 and older (as estrogen levels decline after menopause) (35).

\section{Analysis of drugs triggering transcriptional signatures that mimic the COVIDCs}

Next, we interrogated CMap for the identification of compounds triggering a similar effect on transcription as the one observed in COVID-19 patients, as these compounds could in principle potentiate the severity of the hypercytokinemia. Before discussing the effect of specific compounds, we adapted the Gene Set Enrichment Analysis (GSEA) to conduct a "drug GSEA" in order to identify compound classes that mimic the $\operatorname{COVID}^{\mathrm{CS}}(36)$. Consistent with the analysis from genetic perturbations, PKC activating drugs were the most significantly enriched class, followed by "ATPase inhibitors", "Topoisomerase Inhibitors", "Insulin sensitizers" and "CDC inhibitors" (Fig. $\mathbf{2 A}, \mathbf{B})$. Interestingly, and in relation to insulin, a recent study has found that the triglyceride and glucose index (TyG) correlates with the severity and mortality of COVID-19 patients, which builds upon epidemiological observations that reveal obesity as a risk factor among COVID-19-related fatalities (37). If we restrict our CMap analysis to medically approved drugs, only Topoisomerase inhibitors showed a significant enrichment as drugs mimicking the COVID ${ }^{C S}$, although the absence of other compound classes from this list can be influenced by low number of clinically available drugs in other pathways (Fig. 2C).

In what regards to specific drugs that could potentiate the cytokine response in COVID-19 patients, three of the top compounds were PKC activators: prostratin, phorbol-12-myristate-13-acetate (PMA) and ingenol (Fig. 2D and Table S5). The hydroxychloroquine-related antimalarial drug (mefloquine) was also found among the 
top compounds and was in fact the first compound from the list when the analysis was restricted to drugs already approved for medical use (Fig. 2E and Table S6). We find this of potential interest given the debate that has been around the usefulness of hydroxycloroquine for the treatment of COVID-19, which was questioned by a recent registry analysis of data from 671 hospitals that concluded that the drug increases mortality in COVID-19 patients (38) (although this very study is also now being questioned (39)).

\section{Analysis of drugs triggering transcriptional signatures opposed to the COVIDCS}

As mentioned in the introduction, one of the initial ideas behind the development of CMap was to identify drugs that trigger transcriptional responses that negatively correlate with those associated to a given disease, in order to identify potential new therapies through drug repurposing (24). Based on this concept, we next interrogated CMap for drugs that trigger signatures opposed to the COVIDCS. Drug GSEA analyses identified glucocorticoid receptor agonists as the most significantly compounds that trigger a signature opposed to the COVID ${ }^{\mathrm{CS}}$, followed by Vitamin $\mathrm{K}$ antagonists and MEK inhibitors (Fig. 3A). Furthermore, and in addition to glucocorticoid receptor agonists, the analysis of biological pathways (CMap classes) with signatures negatively correlating to the $\mathrm{COVID}^{\mathrm{CS}}$ also identifies drugs in clinical trials like JAK inhibitors $(17,18)$ (Fig. 3B and Table S7). Other significantly enriched CMap classes include loss-of-function of $\mathbf{S 1 0 0}$ calcium-binding proteins and inhibitors of Bromodomains, Leucine-rich repeat kinases (LRRK) or the MEK kinase.

The connection with COVID-19 of LRRKs, Bromodomains or S100 calcium-binding proteins is unclear at this point. However, we believe that the potential effects of MEK inhibitors in counteracting the cytokine response seem of interest for a number of reasons. First, because even if MEK inhibitors have been mainly developed as antineoplastic agents, numerous studies support that targeting MEK limits cytokine responses in several contexts like graft-vs-host disease, cerebral ischemia, activation of $T$ cells by superantigens or influenza $A$ infection (40-45). Second, because MEK inhibition has been previously shown to inhibit replication of other coronaviruses such as the mouse hepatitis virus (MHV) (46). Third, because besides MEK, several 
compounds with the highest scores of opposing signatures to the COVIDCS are inhibitors of other factors from the same signaling route (MEK, RAF, EGFR, MAPK) (Figure 3C). And, finally, because as far as we can tell inhibition of this signaling route is currently not being explored as a potential therapy in the context of COVID-19 and its associated CS.

Besides compound classes, among specific drugs with associated signatures that negatively correlate with COVID ${ }^{\mathrm{CS}}$ we found compounds known to limit inflammatory responses including the IKK inhibitor TPCA-1, which inhibits NFkB signaling and reduces acute lung injury (47) (Fig. 3D and Table S8). If the analysis was restricted to medically approved compounds the list was led by compounds like the antidepressant phensuximide or the anticoagulant warfarin (Fig. 3E and Table S9), for which the relationship with inflammation is unclear at this point.

\section{Genetic perturbations related to the COVID-19 CS transcriptional signature}

While the previous analyses were performed on a transcriptional signature defined from COVID-19 patients, this was not explicitly mentioned to be obtained from the severe patients undergoing a CS. In order to define a transcriptional signature specifically related to the CS, we used data from another manuscript, where the BALF transcriptome of a COVID-19 patient suffering from a CS was compared to that of healthy individuals or a milder case of the disease (signature available in Table S1; CSs) (48). Consistent with our previous findings on the COVIDCS, NFKB and PKC signaling were the CMap classes most significantly similar to the CS (Fig. 4A and Table S2). Furthermore, IL1 (IL1R1) or TNF $\alpha$ receptors (TNFRS10A and TNFRSF1A) and IFN-response genes (IRF2, IFR5, IFI16) were once again found among the top list of factors that, when overexpressed, trigger a transcriptional signature similar to the CSs (Fig. 4B and Table S3). GO analyses from the top hits among overexpressed factors correlating to the CSs further identified inflammation- and cytokine productionrelated pathways amongst the most significantly enriched ones (Fig. 4C). Interestingly, pathways related to Vitamin D metabolism and angiotensin-activated signaling were also significantly enriched among those that mimic the CSs, both of which have been linked to the severity of COVID-19 $(49,50)$. As for genes that, when 
downregulated, would trigger a transcriptional signature similar to the CSs, this list also showed a significant overlap with that resulting from the COVIDCS analyses (Fig. 4D and Table S4, compare to Fig. 1C). Importantly, GO analyses found once again an enrichment of pathways related to female hormone signaling among those linked to genes with signatures that negatively correlate with the CSs (Fig. 4E), further reinforcing the idea that the anti-inflammatory properties of estrogens might be behind the gender differences found on the severity of COVID-19. We also note an enrichment in pathways related to cholesterol metabolism, which together with the fact that "negative regulation of fat cell proliferation" was found in the corresponding COVIDCS analysis, could be related to the role of obesity as a comorbidity factor for COVID-19 (37).

\section{Identification of drugs triggering transcriptional signatures mimicking the CSs}

We next looked into compounds eliciting a transcriptional signature that positively correlates to the CSs. Despite the disparity of the datasets used, the results from these analyses were also remarkably concordant with our previous findings on the COVID ${ }^{\mathrm{CS}}$. Drug GSEA found PKC activating drugs followed by "ATPase inhibitors" and "Apoptosis Stimulants" as compound classes that significantly correlate with the CSs (Fig. 5A,B); whereas only Topoisomerase inhibitors showed a significant enrichment if medically approved drugs were exclusively analyzed (Fig. 5C). Regarding the potential aggravating effects of topoisomerase inhibitors on the COVID-19 CS, we believe that this is most likely related to Topoisomerase-II. In fact, Topoisomerase-I activity has been found to mediate the expression of inflammatory genes (51). In contrast, Topoisomerase-II inhibition triggers the expression of inflammatory cytokines through activation of cGAS-STING innate immune sensing of cytoplasmic DNA (52).

Regarding specific drugs that could potentially aggravate the CS, and besides PKC activators (ingenol, PMA and prostratin), the histone methyltransferase inhibitor chaetocin has also been shown to boost cytokine production and inflammation (53, 54) (Fig. 5D and Table S5). Interestingly, if we focused on drugs already approved for medical use, the compound which signature most resembled the $\mathrm{CS}^{\mathrm{s}}$ was the ACE inhibitor fosinopril, which is of relevance given the debate on the potential aggravating 
effects of antihypertensive ACE inhibitors in the context of COVID-19 (Fig. 5E and Table S6) (55). Unexpectedly, several drugs used for the treatment of viral or bacterial infections such as nitazoxanide, amantadine or primaquine were also found among approved compounds that trigger a signature similar to the CS . Finally, another drug whose signature mimics the CSs is disulfiram (Antabuse), which is used for the treatment of alcohol abuse disorders (currently used by more than 200,000 patients in the USA (https://www.addictioncenter.com/alcohol/disulfiram/)). While how disulfiram could be related to the CS is at this point unclear, we want to note that, among other targets, this compound is a potent in vitro inhibitor of Topoisomerases (56).

\section{Identification of drugs triggering transcriptional signatures opposing the CSs}

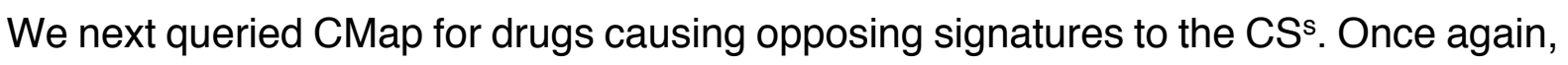
the general results from these analyses were highly consistent with those from the COVIDCS. For instance, drug GSEA analyses yielded glucocorticoid receptor agonists as significantly enriched (Fig. 6A,B). Besides glucocorticoid receptor agonists, the analysis of CMap classes negatively correlating to the CSs again identified inhibitors of Bromodomains, Leucine-rich repeat kinases (LRRK), JAK kinases, V Type ATPases or the MEK kinase (Fig. 6C and Table S7). Remarkably, all compound classes that have been already proposed for the treatment of the COVID-19 CS including corticosteroids, JAK and HMGCR inhibitors triggered transcriptional signatures that negatively correlated with the $\mathrm{CS}^{\mathrm{s}}$, supporting the strength of our approach $(15,57,58)$ (Fig. 6D and Table S7).

The analysis yielded more subtle and interesting differences with the previous COVIDCS analysis when we looked into specific compounds with signatures opposite to the CSs (Fig. 6E and Table S8). Among the top of this list there were several compounds reported to limit inflammatory responses such as treprostinil, a prostacyclin analog that blocks NFkB nuclear translocation in human alveolar macrophages (59); the antidepressant bupropion, which was shown to lower TNF $\alpha$ and IFN $\gamma$ production in mice (60); the IKK inhibitor TPCA-1 which, as mentioned, was shown to inhibit NFkB signaling and to reduce acute lung injury (47); and the farnesyltransferase inhibitor tipifarnib, which presents anti-inflammatory properties 
(61). When the analysis was restricted to drugs approved for medical use (Fig. 6F and Table S9), some of the compounds like niacin (vitamin B3) have already been proposed for the treatment of the CS in COVID-19 patients (62), and others like the calcium channel blocker verapamil reduce lung injury in mice after LPS injections, which triggers a CS-related pathology (63). Noteworthy, a recent study of drugs that could be repositioned for the treatment of COVID-19 based on interactome analyses also identified several compounds that we identify here including verapamil or inhibitors of Bromodomains and RAF kinases (64).

To end, we tried to investigate the potential mechanism by which the antidepressant phensuximide or the vitamin $\mathrm{K}$ antagonist warfarin could counteract the $\mathrm{CS}$, as these two compounds were identified as top hits among medically approved drugs in both the COVID ${ }^{\mathrm{CS}}\left(1^{\text {st }}\right.$ and $2^{\text {nd }}$ hit) and $\mathrm{CS}^{S}$ ( $1^{\text {st }}$ and 4 th) analyses. To this end, we took advantage of a novel bioinformatic tool for the identification of drugs capable of modulating the immune system named DREIMT (http://www.dreimt.org/). DREIMT integrates 4,694 drug transcriptional signatures, with 2,697 manually curated immune gene expression signatures, allowing the identification of novel drug-immune relationships. As expected, DREIMT analyses confirmed that most compounds negatively correlated to the $\mathrm{CS}^{S}$ had transcriptional signatures related to antiinflammatory immune signatures (e.g. treprostinil, tipifarnib and TCPA-1). Remarkably, the transcriptional signatures associated to phensuximide and warfarin were also inversely correlated to inflammation, particularly in macrophages, helping to understand the potential effect of these drugs in counteracting a CS (Table S10). Altogether these analyses have allowed for a better understanding of the transcriptional perturbations associated to COVID-19 and provided a hypothesis for compound classes or specific drugs that could be investigated in the context of the CSs triggered by viral infections.

\section{A CMap analysis of the COVID-19 CS based on single-cell RNAseq data}

Finally, and in order to complement our analyses on whole transcriptomic data, we benefited from a recent study where Liao et al. have reported single-cell RNA-seq data from BALF samples obtained from healthy individuals and COVID-19 patients with either mild disease or ARDS and CS symptoms (65). Their study found that in patients 
undergoing the CS resident FABP4+ alveolar macrophages in the lung are replaced by an overwhelming infiltration of monocyte derived FCN1+ macrophages with an inflammatory gene expression profile. Using transcriptomic data from the lunginfiltrating macrophages analyzed in this study (6 patients), we defined a new transcriptional signature associated to the COVID-19 CS (CSs[sc]) (Table S1), which we then used for CMap analyses.

Regarding transcriptional signatures related to genetic perturbations, NFאB and PKC activation were again the CMap classes with the highest similarity scores to the CS $[\mathrm{sc}]$ (Fig. 7A and Table S2). Moreover, and consistent with our previous analyses, the genes that, when overexpressed, triggered signatures most similar to the CSs[sc] included TNF receptors and IFN-response genes (Fig. 7B and Table S3). In what regards to compounds, drug GSEA analyses found again PKC activators as a significantly enriched compound class that triggers a transcriptional signature mimicking the CSs[sc], together with proteasome, HDAC or HSP inhibitors (Fig. 7C,D). In striking contrast to whole transcriptomic analyses, in this case glucocorticoid receptor agonists were found as potentially stimulating the $\mathrm{CS}$ [sc]. However, we should note that glucocorticoids are known to have cell-type specific effects and specifically in macrophages, where this signature has been defined, they have been shown to promote cytokine release (66). Nevertheless, and in agreement with our previous analyses of the COVIDCS and CS ${ }^{S}$, the top of the list for similarity scores of individual compounds included the same three PKC activators (prostratin, PMA and ingenol) and the histone methyltransferase inhibitor chaetocin, as well as several others (Fig. 7E and Table S5). By restricting the analysis to medically approved compounds, the list is also remarkably similar to our findings made on whole BALF transcriptomics, and again included disulfiram, menadione, amsacrine and nilotinib, as well as multiple topoisomerase inhibitors (amsacrine, teniposide, irinotecan, SN38) (Fig. 7F and Table S6).

Finally, we searched for compounds signatures that negatively correlate with the $\mathrm{CS}^{\mathrm{s}}[\mathrm{sc}]$. Interestingly, the top of this list once again included MEK inhibitors, together with loss of function of aminoacyl-tRNA synthetases and inhibitors of the reverse 
transcriptase, bacterial $30 \mathrm{~S}$ ribosome, PKA and glycogen synthase kinase (Fig. 8A and Table S7). Among specific compounds that elicit transcriptional signatures opposed to the CSs[sc] the top hits included compounds not identified in whole transcriptome analyses and that could potentially be related to counteracting inflammation (Fig. 8B and Table S8). These included the estrogen estriol, relevant in the context of the above mentioned gender-related differences in COVID-19 mortality; the dopamine receptor agonist fenoldopam, previously shown to reduce the severity of LPS-induced lung-injury and cytokine release in mice (67); the CDK inhibitor indirubin, known to reduce the LPS-induced cytokine expression in macrophages (68, $69)$ and to decreased influenza virus derived lung damage $(70,71)$; the Cytochrome P450 inhibitor metyrapone, found to reduce cytokine production in the context of inflammation (72); and the Calcium channel blocker bepridril and Myosin light chain kinase inhibitor ML-7 which have also been reported to present anti-inflammatory roles $(73,74)$. From this list of compounds only fenoldopan, bepridil, metyrapone and estriol are already approved for medical use (Fig. 8C and Table S9).

The top hits among clinically available drugs with a signature negatively correlating to the CSs[sc] also included the RAF inhibitor vemurafenib and the antibiotic doxycycline. For the reasons mentioned above, we are particularly intrigued by the potential of MAPK/MEK/RAF inhibitors in the context of the COVID-19 CS, which became evident in all of our 3 analyses (40-46). As for doxycycline, and besides its usefulness as an antibiotic that might combat bacterial infections triggering pneumonia in COVID-19 patients, doxycycline is also an anti-inflammatory agent (75) and several studies have already suggested its use to combat the COVID-19 CS $(76,77)$. Further supporting these conclusions, drug GSEA analysis revealed MEK inhibitors and "Bacterial cell wall synthesis inhibitors", together with PI3K inhibitors (Fig. 8D,E) as the most significantly enriched classes of compounds with a negatively correlating transcriptional signature to the CSs[sc]. Collectively, these analyses helped to identify compound classes and specific compounds that could potentially revert the transcriptional response associated to the COVID-19 CS. 


\section{CONCLUSION}

We here present our results using large-scale public repositories of transcriptional signatures and bioinformatics tools to identify compounds and genetic perturbations that trigger a transcriptional response similar or opposite to that observed in severe patients of COVID-19 undergoing a CS. We are aware of the caveats of the limited data available to perform these studies at this point, which should get stronger as more transcriptomic data from these patients becomes available. We find nevertheless remarkable the large degree of overlap that we found in our analyses, despite using three different datasets and two different models (whole tissue vs single-cell) to define our transcriptional signatures. In addition to identifying drugs that are already being used (e.g. glucocorticoid receptor antagonists) or are being tested in clinical trials (e.g. JAK inhibitors) as COVID-19 therapies, our study reveals a potential for drug repositioning of compounds like MAPK/RAF/MEK inhibitors, the antidepressant phensuximide or certain antibiotics for treating the COVID-19-associated CS. We must again remind that, in any case, any therapy based on anti-inflammatory drugs should be restricted to the late and severe stages of COVID-19, as their use could limit the efficacy of the immune system in fighting the infection at earlier stages. Besides identifying compounds that could potentially be of use for treating severe cases of COVID-19, we raise awareness on the possible adverse effects that drugs that are widely used such as topoisomerase inhibitors or the alcohol anti-abuse drug disulfiram might have in potentiating the CS. Finally, our manuscript supports that the genderrelated differences on the severity of COVID-19 might be related to the antiinflammatory properties of female hormones. To facilitate the access to our results, we have generated a unified table containing all the data reported in this study (Table S11). We want to end this manuscript with a clear statement in that by no means we are proposing novel clinical indications for any of these agents. We simply wanted to contribute in the context of the current health crisis and provide a resource that others might find of use in the selection of drugs that could be tested for efficacy in experimental systems of CS. 


\section{METHODS}

\section{Calculation of similarity scores}

Three independent transcriptional signatures defined from COVID-19 patients $\left(\mathrm{COVID}^{\mathrm{CS}}, \mathrm{CS}^{\mathrm{s}}\right.$ and CSs[sc]) were used as inputs to query CMap using its clue.io tool (https://clue.io), in order to obtain similarity scores for the signatures associated to all the perturbagens (compounds, CMap classes and the over-expression or knock-down of genes) available at CMap (23). Similarity scores were downloaded, and results sorted based on their scores and the type of perturbagen. For restricting the analysis to medically approved drugs, we used drug information available at CMap. Except for simple filtering we used R version 3.6.3 (2020-02-29) for our computational analyses.

\section{Gene ontology (GO)}

For GO analyses, a list of genes in which their perturbation (overexpression or knockdown) led to a similarity score $>95$ with the COVID-19-associated signatures was used as input in "The Gene Ontology Resource", release 2020-04-23 (http://geneontology.org/) (78-80).

\section{Drug “Gene Set Enrichment Analysis" (GSEA)}

We adapted Gene Set Enrichment Analysis (GSEA) to enable enrichment analyses of "drug classes" based in their mechanism of action (MOA), for which we used the GSEA method implemented in the $R$ package fgsea (36).

\section{DREIMT analyses}

The DREIMT database query tool (http://dreimt.sing-group.org/) was used to search for similarities between the transcriptional signatures triggered by specific compounds and those associated to immunological processes. Similarity scores were downloaded, and results sorted and filtered based on their scores (scores $>90$ or <90 ). 


\section{ACKNOWLEDGMENTS}

The authors want to help Drs. Ignacio Perez de Castro and Andres Joaquin LopezContreras for helpful comments on the manuscript. Work in O.F-C. lab is supported by grants from the Spanish Ministry of Science, Innovation and Universities (RTI2018102204-B-I00 and SAF2017-90900-REDT, co-financed with European FEDER funds) to Oscar Fernandez-Capetillo and by a Ph.D. fellowship from La Caixa Foundation Foundation and the Marie Skłodowska-Curie European Union's Horizon 2020 actions (LCF/BQ/IN17/11620001) to Laura Sanchez-Burgos. CNIO Bioinformatics Unit (BU) is a member of the Spanish National Bioinformatics Institute (INB), ISCIIIBioinformatics platform and is supported by grant PT17/0009/0011, of the Acción Estratégica en Salud 2013-2016 of the Programa Estatal de Investigación Orientada a los Retos de la Sociedad, funded by the ISCIII and European Regional Development Fund (ERDF-EU) and project RETOS RTI2018-097596-B-I00 funded by AEI-MCIU and cofounded by the ERDF-EU. 


\section{REFERENCES}

1. F. Wu et al., A new coronavirus associated with human respiratory disease in China. Nature 579, 265-269 (2020).

2. Anonymous, Novel Coronavirus (2019-nCoV) situation reports. https://www.who.int/docs/default-source/coronaviruse/situation-reports/20200603covid-19-sitrep-135.pdf?sfvrsn=39972feb 2.

3. J. F. Chan et al., Genomic characterization of the 2019 novel human-pathogenic coronavirus isolated from a patient with atypical pneumonia after visiting Wuhan. Emerg Microbes Infect 9, 221-236 (2020).

4. S. Su et al., Epidemiology, Genetic Recombination, and Pathogenesis of Coronaviruses. Trends Microbiol 24, 490-502 (2016).

5. E. de Wit, N. van Doremalen, D. Falzarano, V. J. Munster, SARS and MERS: recent insights into emerging coronaviruses. Nat Rev Microbiol 14, 523-534 (2016).

6. Anonymous, https://ourworldindata.org/mortality-risk-covid.

7. F. Amanat, F. Krammer, SARS-CoV-2 Vaccines: Status Report. Immunity 52, 583-589 (2020).

8. K. Duan et al., Effectiveness of convalescent plasma therapy in severe COVID-19 patients. Proc Natl Acad Sci U S A 117, 9490-9496 (2020).

9. D. L. McKee, A. Sternberg, U. Stange, S. Laufer, C. Naujokat, Candidate drugs against SARS-CoV-2 and COVID-19. Pharmacol Res 10.1016/j.phrs.2020.104859, 104859 (2020).

10. M. Hoffmann et al., SARS-CoV-2 Cell Entry Depends on ACE2 and TMPRSS2 and Is Blocked by a Clinically Proven Protease Inhibitor. Cell 181, 271-280 e278 (2020).

11. V. Monteil et al., Inhibition of SARS-CoV-2 Infections in Engineered Human Tissues Using Clinical-Grade Soluble Human ACE2. Cell 10.1016/j.cell.2020.04.004 (2020).

12. P. Mehta et al., COVID-19: consider cytokine storm syndromes and immunosuppression. Lancet 395, 1033-1034 (2020).

13. J. L. Ferrara, S. Abhyankar, D. G. Gilliland, Cytokine storm of graft-versus-host disease: a critical effector role for interleukin-1. Transplant Proc 25, 1216-1217 (1993).

14. K. J. Huang et al., An interferon-gamma-related cytokine storm in SARS patients. J Med Virol 75, 185-194 (2005).

15. J. R. Tisoncik et al., Into the eye of the cytokine storm. Microbiol Mol Biol Rev 76, 1632 (2012).

16. R. J. Jose, A. Manuel, COVID-19 cytokine storm: the interplay between inflammation and coagulation. Lancet Respir Med 10.1016/S2213-2600(20)30216-2 (2020).

17. M. Zhao, Cytokine storm and immunomodulatory therapy in COVID-19: Role of chloroquine and anti-IL-6 monoclonal antibodies. Int $J$ Antimicrob Agents 10.1016/j.jjantimicag.2020.105982, 105982 (2020).

18. N. E. Ingraham et al., Immunomodulation in COVID-19. Lancet Respir Med 10.1016/S2213-2600(20)30226-5 (2020).

19. Z. Wang et al., Extraction and analysis of signatures from the Gene Expression Omnibus by the crowd. Nat Commun 7, 12846 (2016).

20. A. B. Keenan et al., The Library of Integrated Network-Based Cellular Signatures NIH Program: System-Level Cataloging of Human Cells Response to Perturbations. Cell Syst 6, 13-24 (2018).

21. I. Papatheodorou et al., Expression Atlas: gene and protein expression across multiple studies and organisms. Nucleic Acids Res 46, D246-D251 (2018).

22. J. Lamb et al., The Connectivity Map: using gene-expression signatures to connect small molecules, genes, and disease. Science 313, 1929-1935 (2006).

23. A. Subramanian et al., A Next Generation Connectivity Map: L1000 Platform and the First 1,000,000 Profiles. Cell 171, 1437-1452 e1417 (2017). 
24. A. B. Keenan et al., Connectivity Mapping: Methods and Applications. Annual Review of Biomedical Data Science 2, 69-92 (2019).

25. S. Sinha et al., Systematic cell line-based identification of drugs modifying ACE2 expression. Preprints doi: 10.20944/preprints202003.0446.v1 (2020).

26. Z. Zhou et al., Heightened Innate Immune Responses in the Respiratory Tract of COVID-19 Patients. Cell Host Microbe 10.1016/j.chom.2020.04.017 (2020).

27. M. T. Diaz-Meco, J. Moscat, The atypical PKCs in inflammation: NF-kappaB and beyond. Immunol Rev 246, 154-167 (2012).

28. H. Kim, R. Zamel, X. H. Bai, M. Liu, PKC activation induces inflammatory response and cell death in human bronchial epithelial cells. PLoS One 8, e64182 (2013).

29. J.-M. Jin et al., Gender Differences in Patients With COVID-19: Focus on Severity and Mortality. Frontiers in Public Health 8 (2020).

30. D. K. Biswas, S. Singh, Q. Shi, A. B. Pardee, J. D. Iglehart, Crossroads of estrogen receptor and NF-kappaB signaling. Sci STKE 2005, pe27 (2005).

31. W. E. Naugler et al., Gender disparity in liver cancer due to sex differences in MyD88dependent IL-6 production. Science 317, 121-124 (2007).

32. $\mathrm{H}$. Khalili et al., Oral contraceptives, reproductive factors and risk of inflammatory bowel disease. Gut 62, 1153-1159 (2013).

33. J. L. Nelson, M. Ostensen, Pregnancy and rheumatoid arthritis. Rheum Dis Clin North Am 23, 195-212 (1997).

34. Anonymous, https://www.statista.com/statistics/1105512/coronavirus-covid-19deaths-by-gender-germany/.

35. Anonymous, https://clinicaltrials.gov/ct2/show/NCT04359329?term=estrogen\&cond=covid19\&draw=2\&rank=1.

36. G. Korotkevich, V. Sukhov, A. Sergushichev, Fast gene set enrichment analysis. bioRxiv 10.1101/060012, 060012 (2019).

37. H. Ren et al., Association of the insulin resistance marker TyG index with the severity and mortality of COVID-19. Cardiovasc Diabetol 19, 58 (2020).

38. M. R. Mehra, S. S. Desai, F. Ruschitzka, A. N. Patel, Hydroxychloroquine or chloroquine with or without a macrolide for treatment of COVID-19: a multinational registry analysis. The Lancet https://doi.org/10.1016/S0140-6736(20)31180-6 (2020).

39. T. L. Editors, Expression of concern: Hydroxychloroquine or chloroquine with or without a macrolide for treatment of COVID-19: a multinational registry analysis. The Lancet https://doi.org/10.1016/S0140-6736(20)31290-3 (2020).

40. B. Adler, G. F. Weber, H. Cantor, Activation of T cells by superantigen: cytokine production but not apoptosis depends on MEK-1 activity. Eur J Immuno/ 28, 3749-3754 (1998).

41. M. Kawaguchi et al., Induction of granulocyte-macrophage colony-stimulating factor by a new cytokine, ML-1 (IL-17F), via Raf I-MEK-ERK pathway. J Allergy Clin Immunol 114, 444-450 (2004).

42. Z. Q. Wang, D. C. Wu, F. P. Huang, G. Y. Yang, Inhibition of MEK/ERK 1/2 pathway reduces pro-inflammatory cytokine interleukin-1 expression in focal cerebral ischemia. Brain Res 996, 55-66 (2004).

43. M. B. Miranda, H. Xu, J. A. Torchia, D. E. Johnson, Cytokine-induced myeloid differentiation is dependent on activation of the MEK/ERK pathway. Leuk Res 29, 1293-1306 (2005).

44. R. Pinto et al., Inhibition of influenza virus-induced NF-kappaB and Raf/MEK/ERK activation can reduce both virus titers and cytokine expression simultaneously in vitro and in vivo. Antiviral Res 92, 45-56 (2011). 
45. T. Schrader et al., The clinically approved MEK inhibitor Trametinib efficiently blocks influenza A virus propagation and cytokine expression. Antiviral Res 157, 80-92 (2018).

46. Y. Cai, Y. Liu, X. Zhang, Suppression of coronavirus replication by inhibition of the MEK signaling pathway. J Virol 81, 446-456 (2007).

47. D. Chu, J. Gao, Z. Wang, Neutrophil-Mediated Delivery of Therapeutic Nanoparticles across Blood Vessel Barrier for Treatment of Inflammation and Infection. ACS Nano 9, 11800-11811 (2015).

48. Y. Xiong et al., Transcriptomic characteristics of bronchoalveolar lavage fluid and peripheral blood mononuclear cells in COVID-19 patients. Emerging Microbes \& Infections 9, 761-770 (2020).

49. P. C. Ilie, S. Stefanescu, L. Smith, The role of vitamin D in the prevention of coronavirus disease 2019 infection and mortality. Aging Clin Exp Res 10.1007/s40520-020-015708 (2020).

50. J. Li, X. Wang, J. Chen, H. Zhang, A. Deng, Association of Renin-Angiotensin System Inhibitors With Severity or Risk of Death in Patients With Hypertension Hospitalized for Coronavirus Disease 2019 (COVID-19) Infection in Wuhan, China. JAMA Cardiol 10.1001/jamacardio.2020.1624 (2020).

51. A. Rialdi et al., Topoisomerase 1 inhibition suppresses inflammatory genes and protects from death by inflammation. Science 352, aad7993 (2016).

52. R. D. A. Wilkinson et al., Topoisomerase II inhibitors induce cGAS-STING dependent inflammation resulting in cytokine induction and immune checkpoint activation. Biorxiv https://doi.org/10.1101/764662 (2019).

53. M. F. Li et al., High Glucose Increases the Expression of Inflammatory Cytokine Genes in Macrophages Through H3K9 Methyltransferase Mechanism. J Interferon Cytokine Res 36, 48-61 (2016).

54. T. T. Chen et al., SUV39H1 Reduction Is Implicated in Abnormal Inflammation in COPD. Sci Rep 7, 46667 (2017).

55. L. Fang, G. Karakiulakis, M. Roth, Are patients with hypertension and diabetes mellitus at increased risk for COVID-19 infection? Lancet Respir Med 8, e21 (2020).

56. J. S. Yakisich, A. Siden, P. Eneroth, M. Cruz, Disulfiram is a potent in vitro inhibitor of DNA topoisomerases. Biochem Biophys Res Commun 289, 586-590 (2001).

57. F. Abedi, R. Rezaee, G. Karimi, Plausibility of therapeutic effects of Rho kinase inhibitors against Severe Acute Respiratory Syndrome Coronavirus 2 (COVID-19). Pharmacol Res 156, 104808 (2020).

58. B. Liu, M. Li, Z. Zhou, X. Guan, Y. Xiang, Can we use interleukin-6 (IL-6) blockade for coronavirus disease 2019 (COVID-19)-induced cytokine release syndrome (CRS)? J Autoimmun 10.1016/j.jaut.2020.102452, 102452 (2020).

59. B. Raychaudhuri et al., The prostacyclin analogue treprostinil blocks NFkappaB nuclear translocation in human alveolar macrophages. J Biol Chem 277, 33344-33348 (2002).

60. D. Brustolim, R. Ribeiro-dos-Santos, R. E. Kast, E. L. Altschuler, M. B. Soares, A new chapter opens in anti-inflammatory treatments: the antidepressant bupropion lowers production of tumor necrosis factor-alpha and interferon-gamma in mice. Int Immunopharmacol 6, 903-907 (2006).

61. X. Xue et al., Anti-Inflammatory Activity in Vitro and in Vivo of the Protein Farnesyltransferase Inhibitor Tipifarnib. Journal of Pharmacology and Experimental Therapeutics 317, 53 (2006).

62. Y. Shi et al., COVID-19 infection: the perspectives on immune responses. Cell Death Differ 27, 1451-1454 (2020).

63. Z. Song et al., The therapeutic effect of verapamil in lipopolysaccharide-induced acute lung injury. Biochem Biophys Res Commun 517, 648-654 (2019). 
64. D. E. Gordon et al., A SARS-CoV-2 protein interaction map reveals targets for drug repurposing. Nature 10.1038/s41586-020-2286-9 (2020).

65. M. Liao et al., Single-cell landscape of bronchoalveolar immune cells in patients with COVID-19. Nat Med 10.1038/s41591-020-0901-9 (2020).

66. M. D. van de Garde et al., Chronic exposure to glucocorticoids shapes gene expression and modulates innate and adaptive activation pathways in macrophages with distinct changes in leukocyte attraction. J Immunol 192, 1196-1208 (2014).

67. N. B. Bone, Z. Liu, J. F. Pittet, J. W. Zmijewski, Frontline Science: D1 dopaminergic receptor signaling activates the AMPK-bioenergetic pathway in macrophages and alveolar epithelial cells and reduces endotoxin-induced ALI. J Leukoc Biol 101, 357365 (2017).

68. A. S. Babcock, A. L. Anderson, C. D. Rice, Indirubin-3'-(2,3 dihydroxypropyl)oximether (E804) is a potent modulator of LPS-stimulated macrophage functions. Toxicol Appl Pharmacol 266, 157-166 (2013).

69. T. Qi, H. Li, S. Li, Indirubin improves antioxidant and anti-inflammatory functions in lipopolysaccharide-challenged mice. Oncotarget 8, 36658-36663 (2017).

70. C. Jie et al., Indirubin, a bisindole alkaloid from Isatis indigotica, reduces H1N1 susceptibility in stressed mice by regulating MAVS signaling. Oncotarget 8, 105615105629 (2017).

71. H. H. Kwok et al., Anti-inflammatory effects of indirubin derivatives on influenza A virusinfected human pulmonary microvascular endothelial cells. Sci Rep 6, 18941 (2016).

72. D. C. Peruzzo et al., Evidence that metyrapone in the presence of inflammation modulates cytokine mRNA expression. Cytokine 52, 184-189 (2010).

73. A. Fujiki, T. Sakamoto, K. Nishida, K. Mizumaki, H. Inoue, Relation of Interleukin- 6 and C-Reactive Protein Levels to Sinus Maintenance After Pharmacological Cardioversion in Persistent Atrial Fibrillation. Journal of Cardiovascular Pharmacology 50 (2007).

74. C. Huang et al., ML-7 attenuates airway inflammation and remodeling via inhibiting the secretion of Th2 cytokines in mice model of asthma. Mol Med Rep 17, 6293-6300 (2018).

75. M. Henehan, M. Montuno, A. De Benedetto, Doxycycline as an anti-inflammatory agent: updates in dermatology. J Eur Acad Dermatol Venereol 31, 1800-1808 (2017).

76. C. Conforti, R. Giuffrida, I. Zalaudek, N. Di Meo, Doxycycline, a widely used antibiotic in dermatology with a possible anti-inflammatory action against IL-6 in COVID-19 outbreak. Dermatol Ther 10.1111/dth.13437, e13437 (2020).

77. A. Farouk, S. Salman, Dapsone and doxycycline could be potential treatment modalities for COVID-19. Med Hypotheses 140, 109768 (2020).

78. M. Ashburner et al., Gene ontology: tool for the unification of biology. The Gene Ontology Consortium. Nat Genet 25, 25-29 (2000).

79. H. Mi, A. Muruganujan, D. Ebert, X. Huang, P. D. Thomas, PANTHER version 14: more genomes, a new PANTHER GO-slim and improvements in enrichment analysis tools. Nucleic Acids Res 47, D419-D426 (2019).

80. The Gene Ontology Consortium, The Gene Ontology Resource: 20 years and still GOing strong. Nucleic Acids Research 47, D330-D338 (2018). 
A

\section{CMAP CLASSES CORRELATING TO COVID ${ }^{\text {CS }}$}

NFKB Activation GOF-

PKC activato

Retinoid receptor agonist

Heat shock $70 \mathrm{kDa}$ proteins LOF

IKK inhibito

Topoisomerase inhibitor-

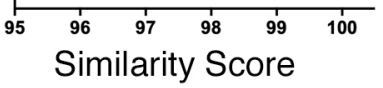

B

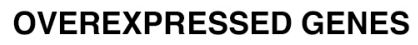
CORRELATING TO COVID ${ }^{\mathrm{SS}}$

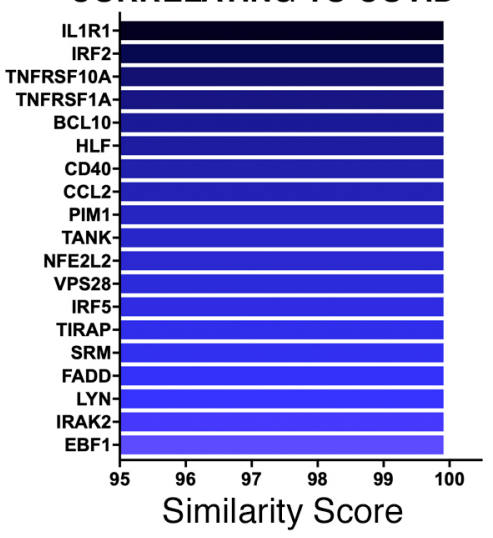

D

\section{GICAL PATHWAYS ENRICHED WITH A NEGATIVE CORRELATION TO COVID19 ${ }^{\text {CS }}$}

\begin{tabular}{|l|c|c|}
\hline \multicolumn{1}{|c|}{ GO TERM } & Fold Enrichment & FDR \\
\hline negative regulation of fat cell proliferation (GO:0070345) & 30,3 & 0,02 \\
\hline thelarche (GO:0042695) & 30,3 & 0,02 \\
\hline mammary gland branching involved in thelarche (GO:0060744) & 30,3 & 0,02 \\
\hline development of secondary female sexual characteristics (GO:0046543) & 28,9 & 0,00 \\
\hline development of secondary sexual characteristics (GO:0045136) & 22,4 & 0,01 \\
\hline nucleotide-binding oligomerization domain containing 2 signaling pathway (GO:0070431) & 21,6 & 0,03 \\
\hline atrioventricular canal development (GO:0036302) & 21,6 & 0,03 \\
\hline alkanesulfonate metabolic process (GO:0019694) & 21,6 & 0,03 \\
\hline taurine metabolic process (GO:0019530) & 21,6 & 0,03 \\
\hline SREBP signaling pathway (GO:0032933) & 16,8 & 0,05 \\
\hline histone-serine phosphorylation (GO:0035404) & 16,8 & 0,01 \\
\hline positive regulation of cell fate commitment (GO:0010455) & 16,8 & 0,05 \\
\hline negative regulation of cell migration involved in sprouting angiogenesis (GO:0090051) & 13,5 & 0,02 \\
\hline hormone catabolic process (GO:0042447) & 12,6 & 0,02 \\
\hline error-prone translesion synthesis (GO:0042276) & 12,0 & 0,01 \\
\hline error-free translesion synthesis (GO:0070987) & 11,5 & 0,01 \\
\hline protein heterooligomerization (GO:0051291) & 11,5 & 0,01 \\
\hline
\end{tabular}

DOWNREGULATED GENES CORRELATING TO COVID ${ }^{\text {cs }}$

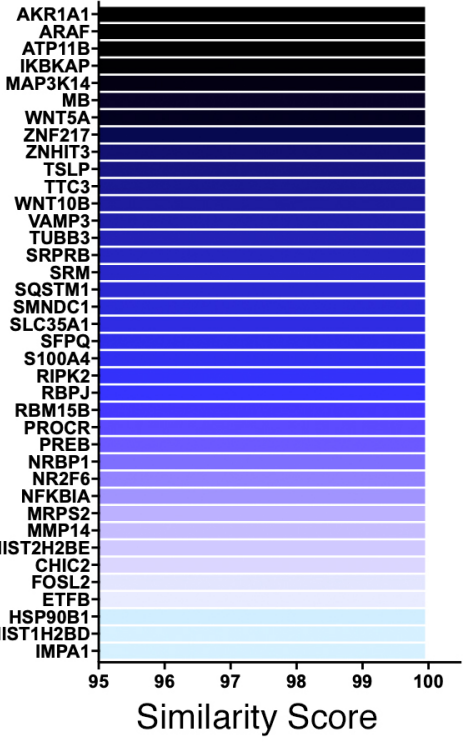


A

\section{B}
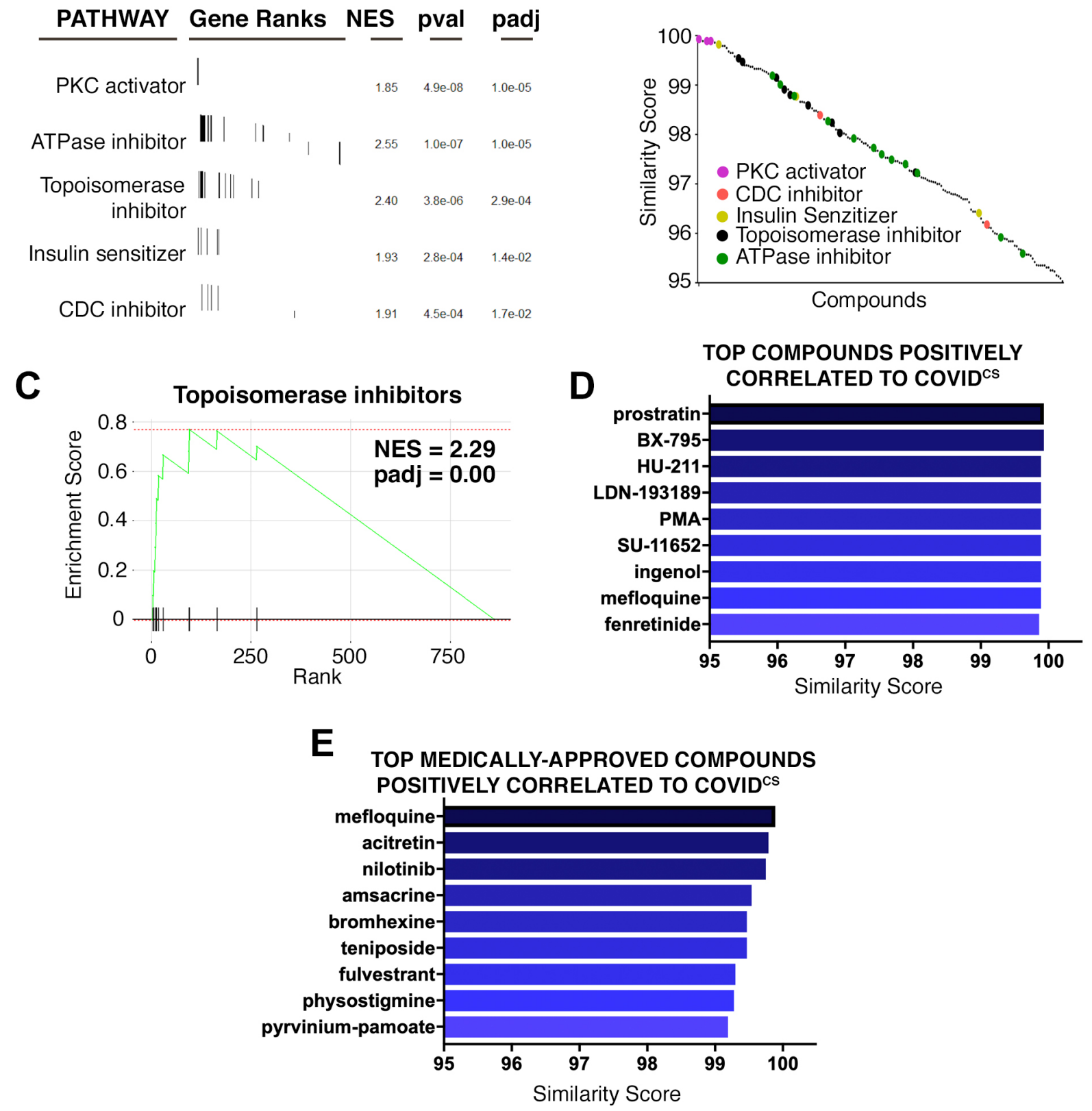
A

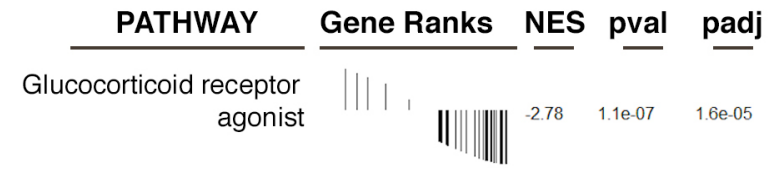

Vitamin $\mathrm{K}$ antagonist

$\mid \begin{array}{lll}-1.87 & 2.0 \mathrm{e}-05 & 1.2 \mathrm{e}-03\end{array}$

MEK inhibitor

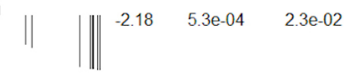

C

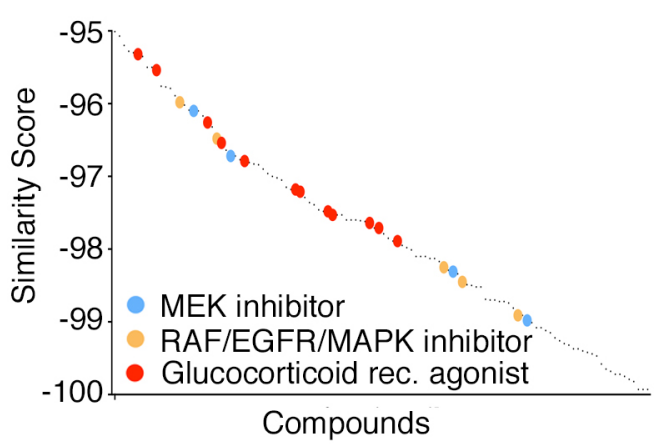

B

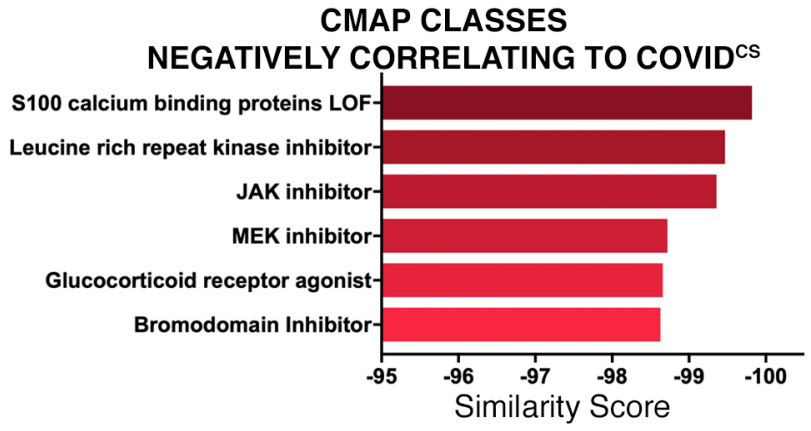

D

\section{TOP COMPOUNDS NEGATIVELY CORRELATED TO COVID ${ }^{\text {CS }}$}

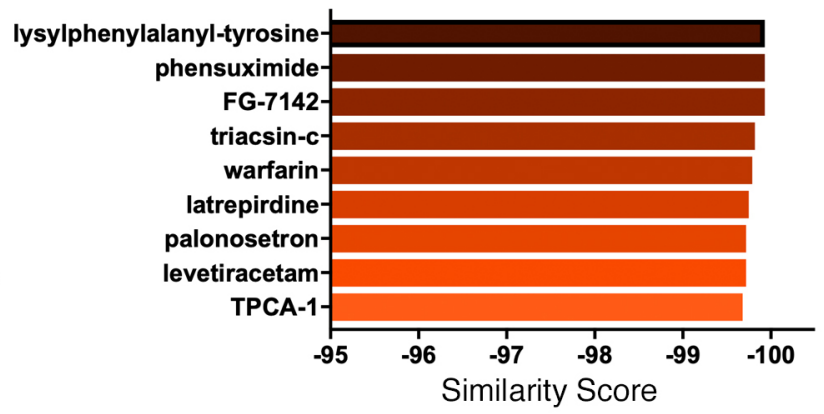

E

\section{TOP MEDICALLY-APPROVED COMPOUNDS} NEGATIVELY CORRELATED TO COVID ${ }^{\mathrm{CS}}$

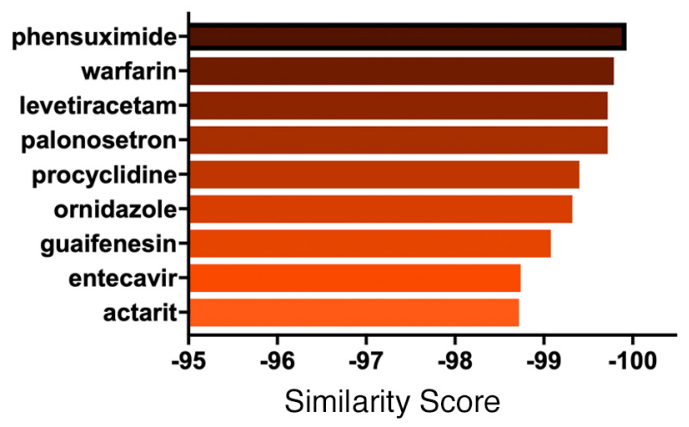


A

CMAP CLASSES CORRELATING TO CS

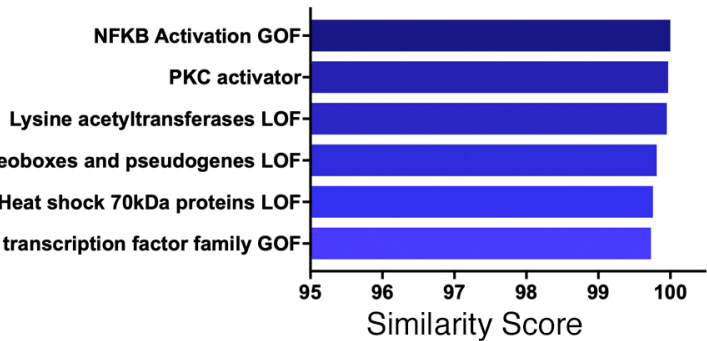

NKL subclass homeoboxes and pseudogenes LOF FOS transcription factor family GOF

Similarity Score

\section{BIOLOGICAL PATHWAYS ENRICHED AMONG GENES WITH A POSITIVE CORRELATION TO CS $^{\text {s }}$}

\begin{tabular}{|l|c|c|}
\hline \multicolumn{1}{|c|}{ GO TERM } & Fold Enrichment & FDR \\
\hline angiotensin-activated signaling pathway involved in heart process (GO:0086098) & $>100$ & 0,01 \\
\hline positive regulation of calcidiol 1-monooxygenase activity (GO:0060559) & $>100$ & 0,00 \\
\hline positive regulation of vitamin D biosynthetic process (GO:0060557) & $>100$ & 0,00 \\
\hline nitric oxide homeostasis (GO:0033484) & 93,3 & 0,01 \\
\hline vitamin D receptor signaling pathway (GO:0070561) & 93,3 & 0,01 \\
\hline positive regulation of sulfur metabolic process (GO:0051176) & 93,3 & 0,01 \\
\hline TRAM-dependent toll-like receptor 4 signaling pathway (GO:0035669) & 93,3 & 0,01 \\
\hline TRAM-dependent toll-like receptor signaling pathway (GO:0035668) & 93,3 & 0,01 \\
\hline positive regulation of vitamin metabolic process (GO:0046136) & 84,0 & 0,00 \\
\hline TRAlL-activated apoptotic signaling pathway (GO:0036462) & 84,0 & 0,00 \\
\hline positive regulation of cell adhesion molecule production (GO:0060355) & 70,0 & 0,02 \\
\hline death-inducing signaling complex assembly (GO:0071550) & 70,0 & 0,00 \\
\hline sequestering of triglyceride (GO:0030730) & 70,0 & 0,02 \\
\hline regulation of calcidiol 1-monooxygenase activity (GO:0060558) & 60,0 & 0,00 \\
\hline positive regulation of T-helper 1 cell cytokine production (GO:2000556) & 56,0 & 0,03 \\
\hline regulation of T-helper 1 cell cytokine production (GO:2000554) & 56,0 & 0,03 \\
\hline regulation of gap junction assembly (GO:1903596) & 56,0 & 0,03 \\
\hline
\end{tabular}

EE

\section{BIOLOGICAL PATHWAYS ENRICHED AMONG GENES WITH A NEGATIVE CORRELATION TO CS $^{\text {s }}$}

\begin{tabular}{|l|r|l|}
\hline \multicolumn{1}{|c|}{ GO TERM } & Fold Enrichment & FDR \\
\hline abortive mitotic cell cycle (GO:0033277) & 50,2 & 0,05 \\
\hline positive regulation of timing of anagen (GO:0051885) & 50,2 & 0,05 \\
\hline Schwann cell proliferation (GO:0014010) & 50,2 & 0,05 \\
\hline negative regulation of oxidative phosphorylation uncoupler activity (GO:2000276) & 50,2 & 0,05 \\
\hline adenine salvage (GO:0006168) & 50,2 & 0,05 \\
\hline nucleotide-binding oligomerization domain containing 1 signaling pathway (GO:0070427) & 50,2 & 0,05 \\
\hline chemorepulsion of dopaminergic neuron axon (GO:0036518) & 50,2 & 0,05 \\
\hline histone H3-T6 phosphorylation (GO:0035408) & 50,2 & 0,05 \\
\hline cholesterol esterification (GO:0034435) & 37,7 & 0,01 \\
\hline sterol esterification (GO:0034434) & 37,7 & 0,01 \\
\hline steroid esterification (GO:0034433) & 37,7 & 0,01 \\
\hline thelarche (GO:0042695) & 30,2 & 0,01 \\
\hline mammary gland branching involved in thelarche (GO:0060744) & 30,2 & 0,01 \\
\hline development of secondary female sexual characteristics (GO:0046543) & 28,7 & 0,00 \\
\hline development of secondary sexual characteristics (GO:0045136) & 27,9 & 0,00 \\
\hline mammary gland involution (GO:006056) & 25,1 & 0,02 \\
\hline chemorepulsion of axon (GO:0061643) & 25,1 & 0,02 \\
\hline
\end{tabular}

B

OVEREXPRESSED GENES CORRELATING TO CS $^{S}$

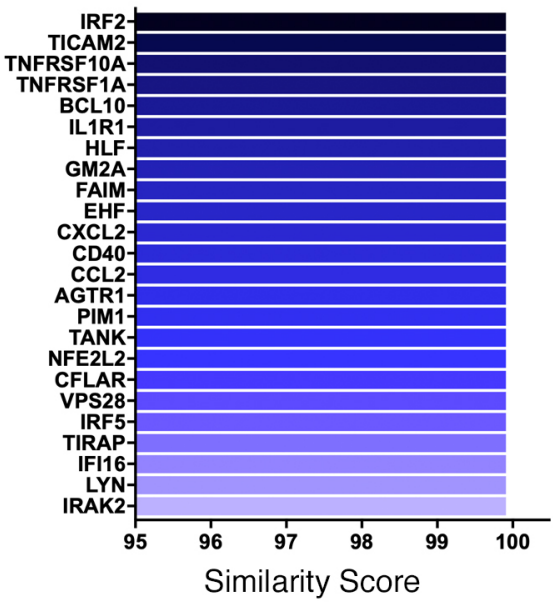

D

DOWNREGULATED GENES CORRELATING TO CS $^{\text {s }}$

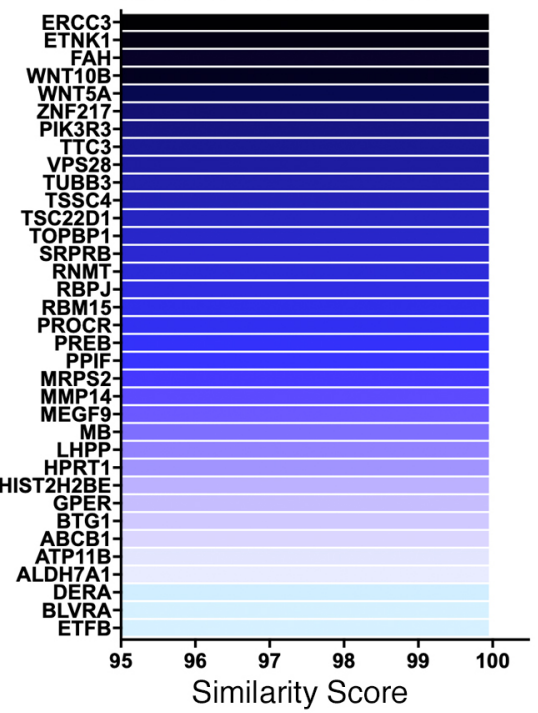


A
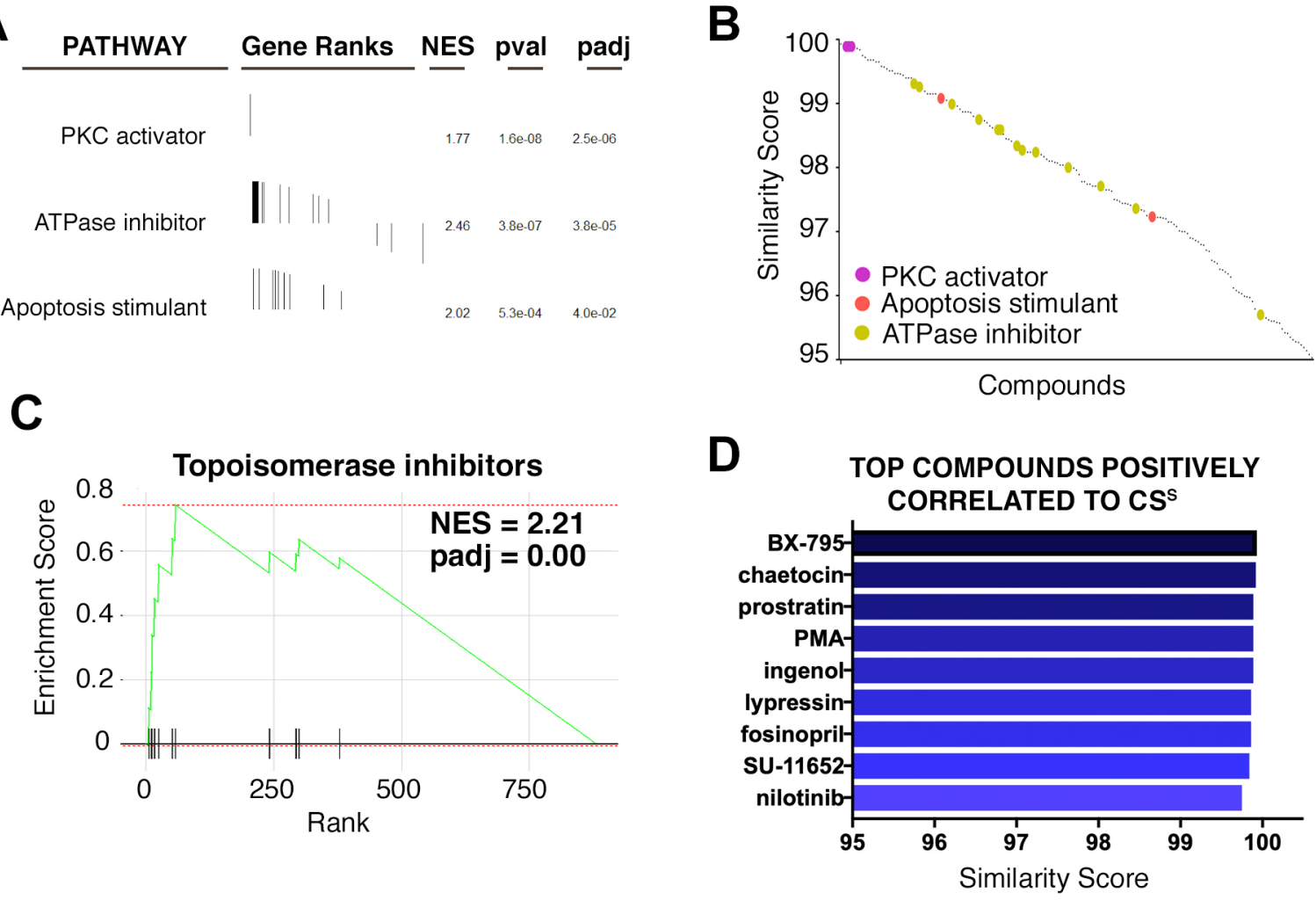

\section{E TOP MEDICALLY-APPROVED COMPOUNDS POSITIVELY CORRELATED TO CS $^{\text {s }}$}

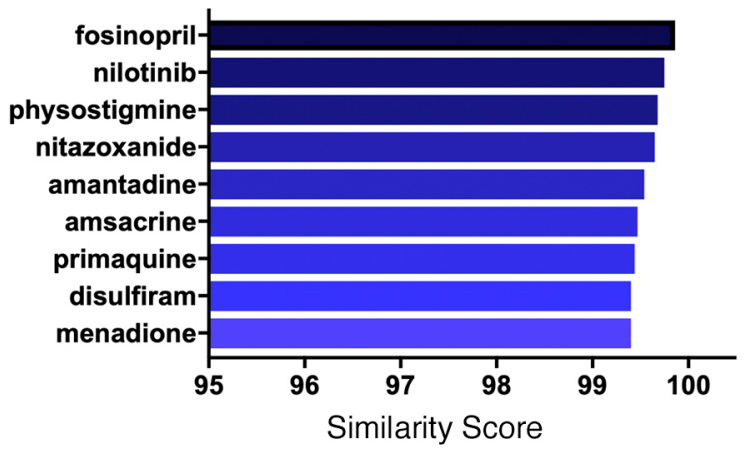



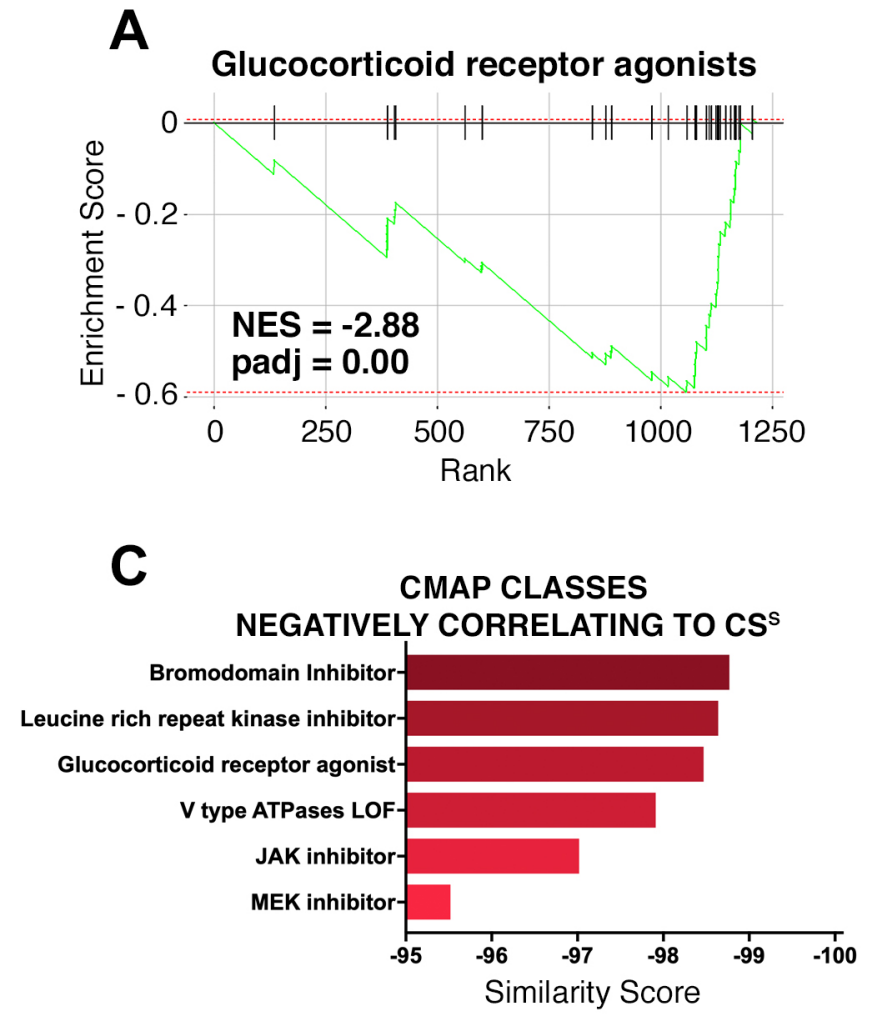

$\mathbf{E}$

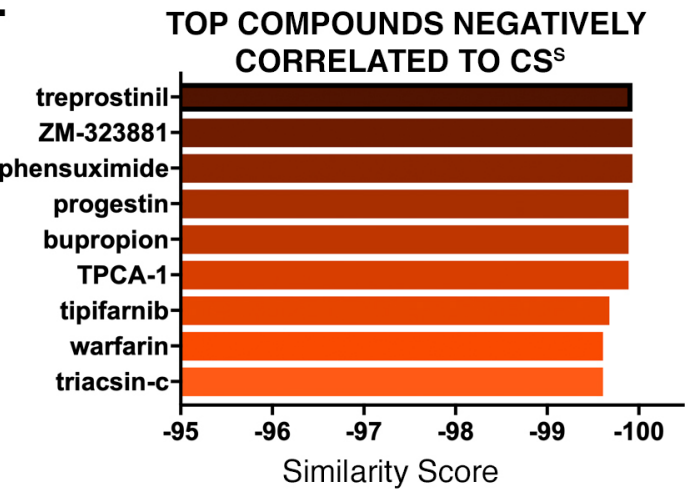

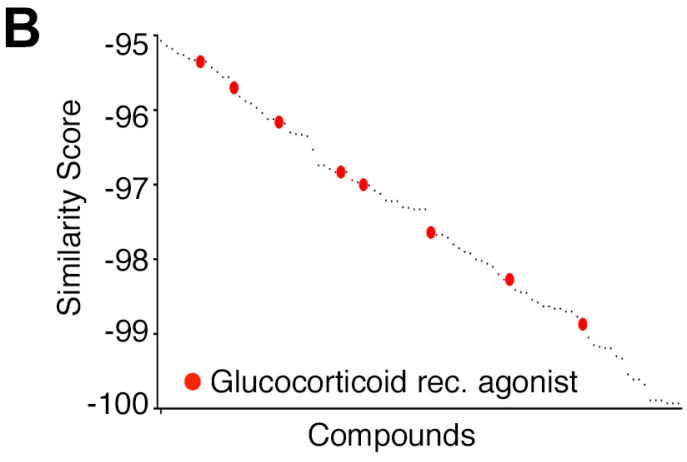

D

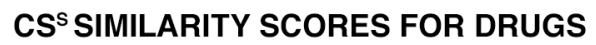
UNDER STUDY FOR THE TREATMENT OF THE COVID-19 CYTOKINE STORM

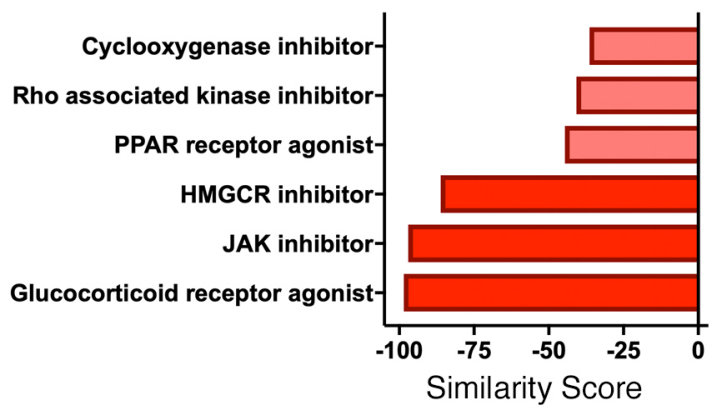

$\mathbf{F}$
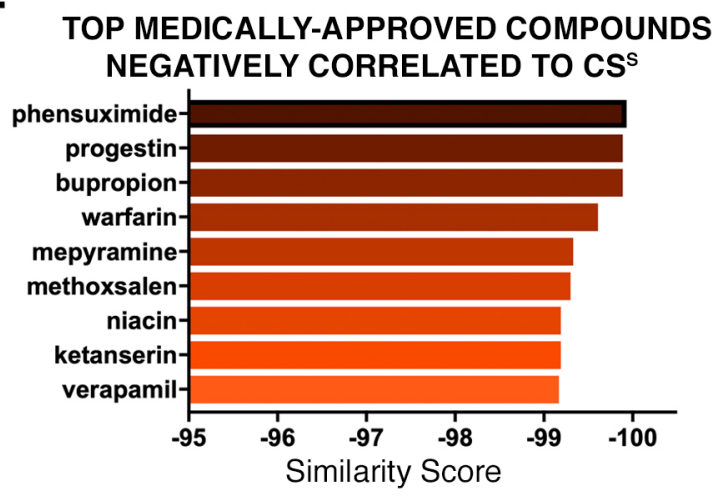


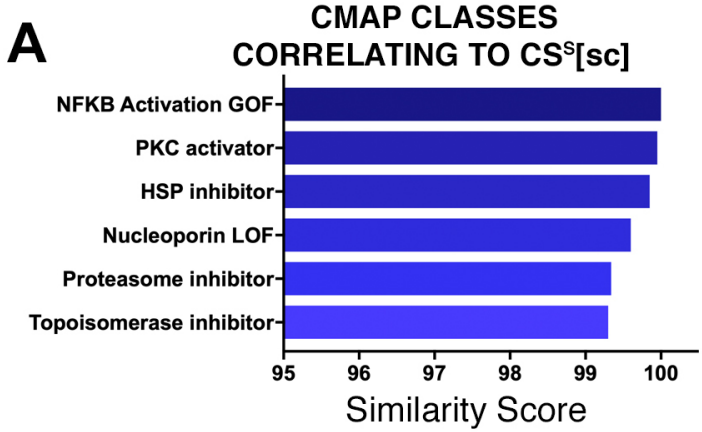

C

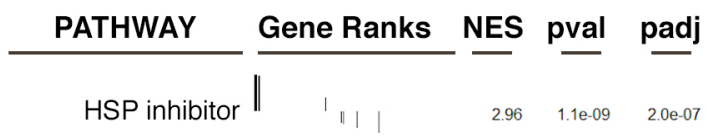

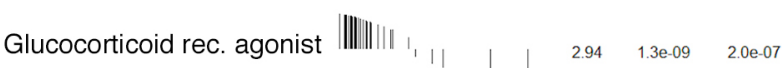

HDAC inhibitor |||||| ||

PKC activator |

|| $\mid \begin{array}{lll}2.77 & 2.9 \mathrm{e}-08 & 2.9 \mathrm{e}-06\end{array}$

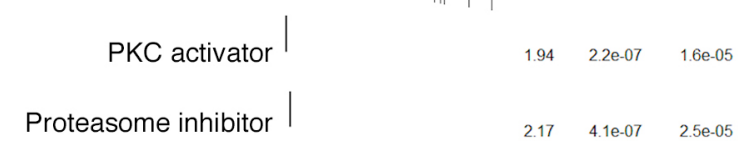

E

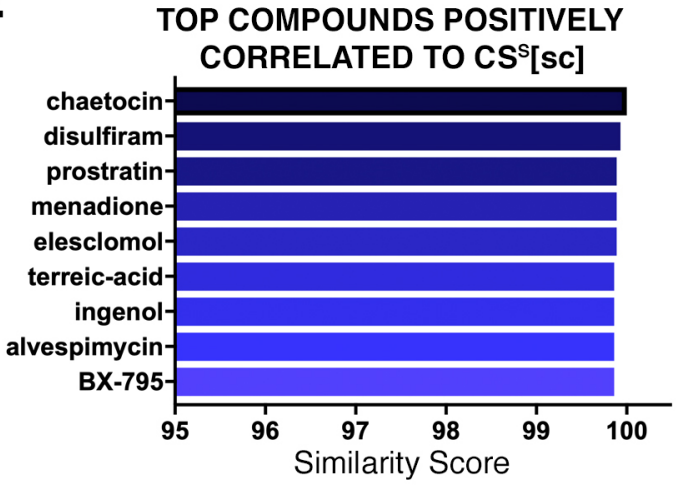

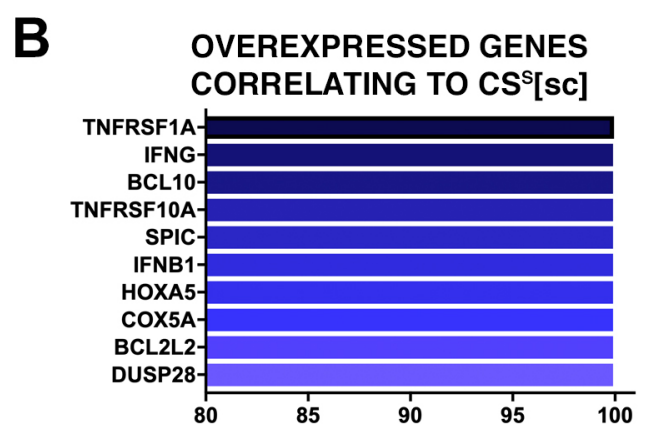

D

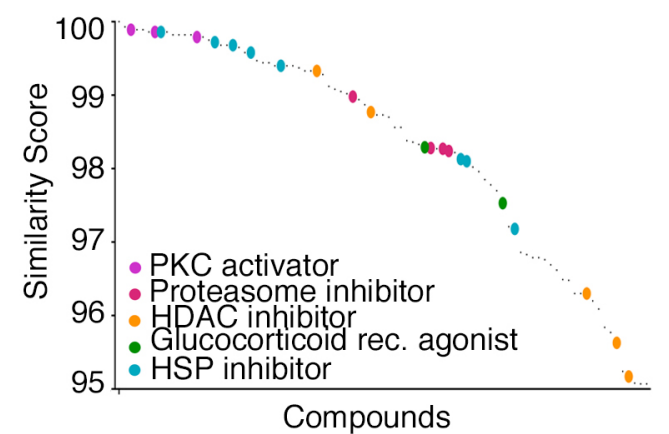

F

TOP MEDICALLY-APPROVED COMPOUNDS POSITIVELY CORRELATED TO $\mathrm{CS}^{\mathrm{S}}$ [sc]

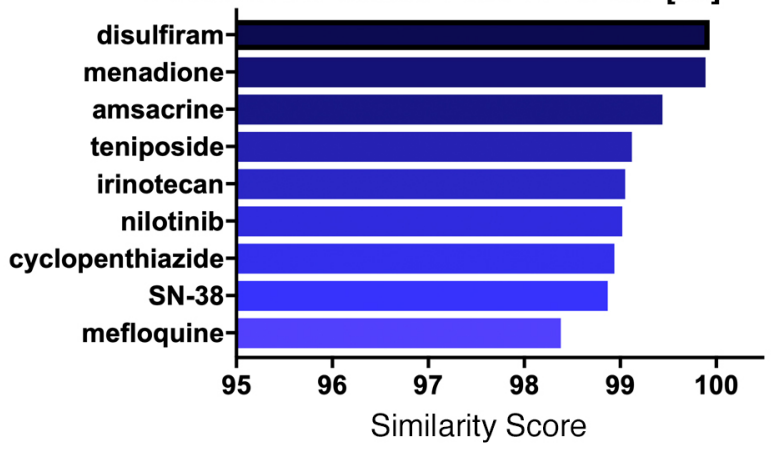


A

CMAP CLASSES NEGATIVELY CORRELATING TO $\mathrm{CS}^{\mathrm{S}}[\mathrm{sc}]$

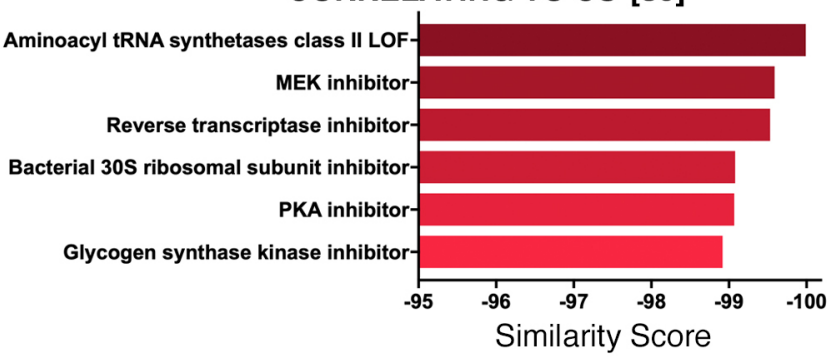

\section{TOP COMPOUNDS NEGATIVELY CORRELATED TO $\mathrm{CS}^{\mathrm{s}}$ [sc]}

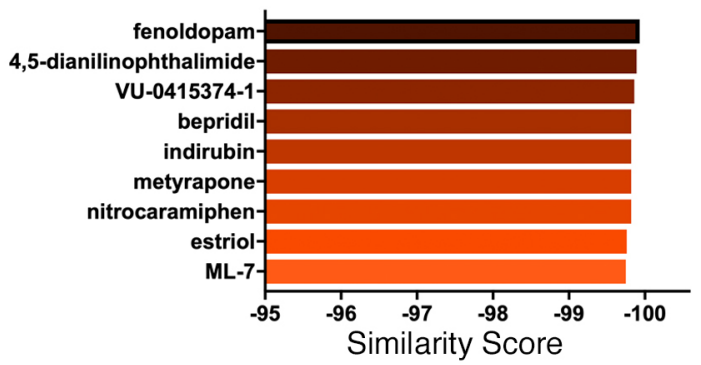

$D$

\section{TOP MEDICALLY-APPROVED COMPOUNDS NEGATIVELY CORRELATED TO CS'[sc]}

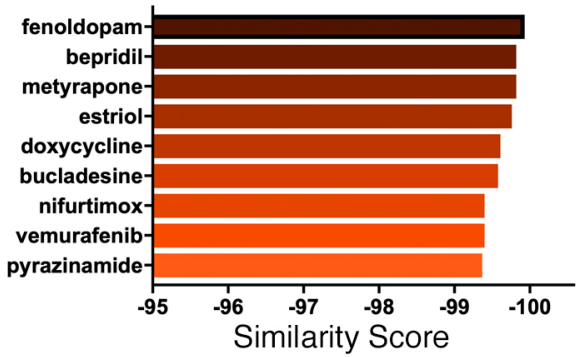

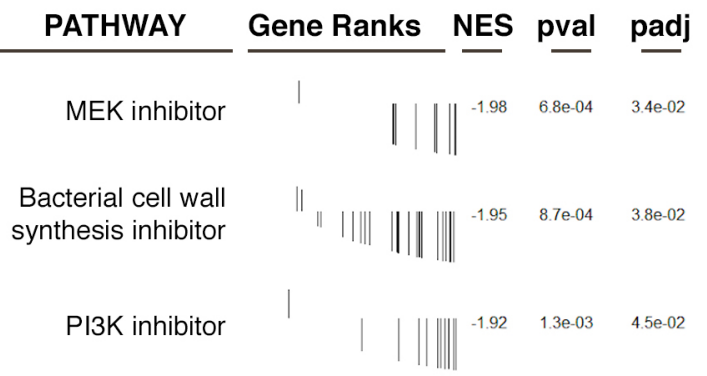
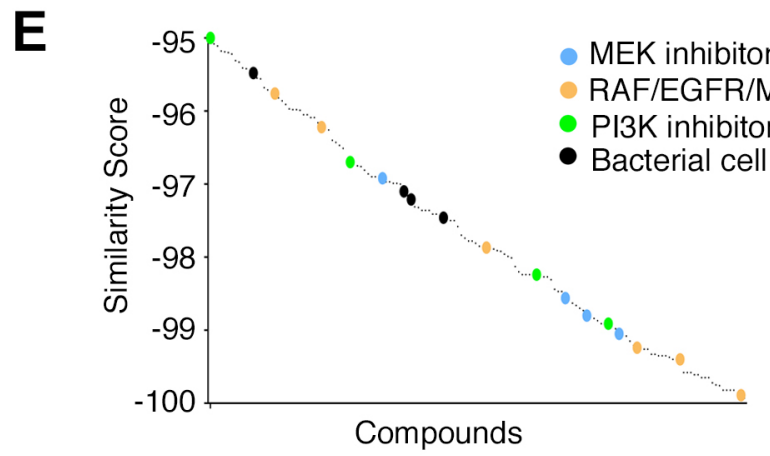